\title{
ORIGINAL ARTICLE Distinguishing the effects of selection from demographic history in the genetic variation of two sister passerines based on mitochondrial-nuclear comparison
}

\author{
C-M Hung ${ }^{1}$ and RM Zink ${ }^{2}$
}

Determining the mechanisms responsible for the distribution of genetic diversity in natural populations has occupied a central role in molecular evolution. Our study was motivated by the unprecedented observation that a widespread Eurasian flycatcher, Ficedula albicilla, exhibited no variation at the mitochondrial DNA (mtDNA) ND2 gene in 75 individuals sampled over a 5000-km distance. In contrast, its sister species, F. parva, had low but considerably higher levels of mtDNA variation. We assessed whether natural selection or demographic factors could explain the absence of mtDNA variation in $F$. albicilla. Eighteen nuclear genes were sequenced to estimate the two species' phylogeographic histories, and for comparison to the mtDNA data. Multilocus coalescence analyses suggested that F. albicilla experienced a population expansion perhaps following a population bottleneck. Simulations based on this demographic history, however, did not replicate the extremely low level of mtDNA variation. Historical range changes based on ecological niche models also failed to explain the observed mtDNA patterns. Neutrality tests (DHEW and ML-HKA) suggested a non-neutral pattern in the mtDNA of $F$. albicilla. We found a transmembrane-skewed distribution of nonsynonymous substitutions between the two species, three of which caused functional change; the results implied that positive selection could have targeted mtDNA. Several lines of evidence support selection rather than demographic history as the main force influencing the patterns of mtDNA variation. Despite the influence of natural selection, many of the phylogeographic inferences derived from mtDNA were robust, including species limits and a high level of gene flow among populations within species.

Heredity (2014) 113, 42-51; doi:10.1038/hdy.2014.9; published online 12 March 2014

\section{INTRODUCTION}

Whether the distribution of genetic variation is best explained by neutral or selective forces has fueled an extended debate in evolutionary biology (Hughes, 2007; Hahn, 2008). The neutral theory predicts that most genetic variants have few or no effects on fitness, and thus genetic variation is a function of mutation rate and population size (Kimura, 1983). Some authors argue that positive (or adaptive) selection is widespread and that the predicted relationship between population size and genetic variation is rarely observed (for example, Hahn, 2008). Many authors have inferred that positive selection influences many genes, particularly mitochondrial DNA (mtDNA; for example, Bazin et al., 2006; Bensch et al., 2006). If true, estimates of phylogeographical history based solely on mtDNA could be biased (Edwards and Bensch, 2009).

Genetic variation at the population level can be influenced by demographic factors, selective factors or both. For example, population bottlenecks can reduce genetic variation through reducing population sizes and facilitating genetic drift (Nei et al., 1975). Similarly, selective fixation of a beneficial mutation can abruptly reduce genetic variation in a gene and genes linked to the targeted gene via hitchhiking (selective sweeps; Maynard-Smith and Haigh, 1974). Although theoretical work for each of these factors is well developed, disentangling the two confounding factors from empirical data remains challenging.

The influence of demographic history should be observed genomewide, whereas selection is more likely to affect different regions of a genome independently (Hudson et al., 1987). Therefore, comparisons of genetic variation across unlinked genes have been used to test for departures from neutrality. However, stochastic coalescence or mutation processes can result in seemingly inconsistent variation among genes even under neutrality (Rosenberg and Nordborg, 2002). Thus, empirical comparisons should take into account such stochasticity to prevent biased inferences. In addition, demographic and selective factors could work in concert to shape the genetic variation of populations or species. Therefore, understanding the confounding effect of demographic history is essential to assess the effect of selection on observed patterns of genetic variation in nature.

mtDNA variation in two sister species of Old World flycatchers, Ficedula albicilla and F. parva, was studied to evaluate the potentially confounding influences of demography and selection in shaping observed genetic variation. Zink et al. (2008) found that 75 out of 75 F. albicilla individuals, sampled across its breeding range spanning around $5000 \mathrm{~km}$, shared the same ND2 haplotype based on entire gene sequences (1041 bp). On the other hand, they found five ND2

${ }^{1}$ Department of Life Science, National Taiwan Normal University, Taipei, Taiwan and ${ }^{2}$ Bell Museum and Department of Ecology, Evolution and Behavior, University of Minnesota, St Paul, MN, USA

Correspondence: Dr C-M Hung, Department of Life Science, National Taiwan Normal University, 88 Ting-Chow Road, Sec 4, Taipei 116, Taiwan.

E-mail: ymwur1@gmail.com

Received 9 September 2013; revised 1 December 2013; accepted 9 January 2014; published online 12 March 2014 
haplotypes among 16 individuals of its sister species, F. parva. This discordant pattern raises the question of whether the different patterns of mtDNA variation are caused by different demographic histories or natural selection. If selection is the driving force, another question is whether selection influences mtDNA directly or indirectly via hitchhiking. In this study, we estimated the demographic histories of the two study species based on 18 nuclear genes, and then used the estimated histories to simulate neutral expectations to test mtDNA diversity for departures from neutrality. Furthermore, we applied neutrality tests, protein structure modeling and ecological niche modeling (ENM) to explore other influences on observed mtDNA variation. Three questions were addressed. First, we examined whether the two sister species experienced different population dynamics. Second, we tested whether the pattern of mtDNA variation departed from neutral expectation by accounting for species' demographic history. Finally, we determined whether positive selection might have eliminated most of the mtDNA variation.

\section{MATERIALS AND METHODS}

Sequence data collection and analyses

ND2 sequence data (1041 bp) for 16 F. parva and 75 F. albicilla (NCBI accession numbers EU326696-326786) were used from the study by Zink et al. (2008) study, in which F. albicilla samples were referred to as F. parva (The two species were considered the same species). The ND2 sequences of one $F$. parva from Sweden were downloaded from GenBank (NCBI accession number GU358799). Zink et al. (2008) used two primer pairs to amplify and sequence ND2 to insure the authenticity of the mitochondrial sequences. We further checked mitochondrial authenticity in two ways: (1) using long PCR to amplify about half the mtDNA genome from ND6 to COI (around $8000 \mathrm{bp}$ ) or a fragment from $16 \mathrm{~S}$ to COI (around $4000 \mathrm{bp}$ ) and then sequencing ND2, and (2) sequencing two other mtDNA genes, Cytb and COI (see Supplementary Materials for the PCR and sequencing protocols for mtDNA). The Cytb (1026 bp) gene was sequenced for $15 \mathrm{~F}$. parva and $25 \mathrm{~F}$. albicilla (Table 1). The Cytb gene of one F. parva from the Czech Republic was downloaded from GenBank (NCBI accession number AJ299689). The COI (1551 bp) gene was sequenced for $16 \mathrm{~F}$. parva and $26 \mathrm{~F}$. albicilla (Table 1).

We sequenced 15 autosomal and two Z-linked introns (Friesen et al., 1999; Shapiro and Dumbacher, 2001; Primmer et al., 2002; Backström et al., 2006, 2008; Kimball et al., 2009) and one exon (Mc1r; MacDougall-Shackleton et al., 2003; Table 1) for variable numbers of individuals. Fourteen allele copies (two allele copies in one individual) of Mc1r were collected for F. parva and 22 allele copies for F. albicilla (Table 1). We collected 13-24 allele copies in the 17 introns for each species (mean $=18.5$ for F. parva and 22.2 for F. albicilla; Table 1). Sample localities are given in Figure 1 and Supplementary Table 1.

The phases of sequences containing indels were sorted manually by subtracting chromatogram peaks upstream of the indel in the reverse primer sequences from the double peaks downstream of the indel in the forward primer sequences; this process was repeated in the alternative direction (SousaSantos et al., 2005). The lengths of indels could also be determined by this approach. Multibase indels were collapsed to single-base polymorphisms for further analyses.

The allelic states of individuals with multiple heterozygous sites but no indels were resolved using PHASE 2.1.1 (Stephens et al., 2001). Homozygous genotypes and genotypes with single heterozygous sites or with indels were set as known alleles to improve the performance of PHASE analyses. The phases of some sites could not be estimated confidently at $90 \%$ posterior probability.

Table 1 Characteristics of the 19 genes (the three mtDNA regions belong to the same linkage group) in this study

\begin{tabular}{|c|c|c|c|c|c|c|c|c|}
\hline Gene & Chr & $L$ & $\mathrm{~N}_{\text {FIPA }}$ & $\mathrm{N}_{\mathrm{FIAL}}$ & $\pi_{\mathrm{FIPA}}$ & $\pi_{\mathrm{FIAL}}$ & Dxy & Indel length \\
\hline ND2 & $\mathrm{Mt}^{\mathrm{a}}$ & 1041 & 17 & 75 & 0.00138 & 0 & 0.06854 & \\
\hline Cyt $b$ & $\mathrm{Mt}^{\mathrm{a}}$ & 1026 & 16 & 25 & 0.00193 & 0.00016 & 0.06069 & \\
\hline \multirow[t]{2}{*}{$\mathrm{COI}$} & $\mathrm{Mt}^{\mathrm{a}}$ & 1551 & 16 & 26 & 0.00092 & 0.00005 & 0.05027 & \\
\hline & & & & Mean & 0.00141 & 0.00007 & 0.05983 & \\
\hline 00132 & 26 & 324 & 20 & 24 & 0.00332 & 0 & 0.00549 & 6 \\
\hline 06635 & 6 & 540 & 20 & 24 & 0.00541 & 0.00749 & 0.01767 & $2,4,5$ \\
\hline 08352 & 5 & 348 & 20 & 24 & 0.00166 & 0.00378 & 0.01468 & 1 \\
\hline 09385 & 20 & 283 & 20 & 22 & 0.00802 & 0.01859 & 0.01397 & $1,1,4,7,7,7$ \\
\hline 11074 & 20 & 376 & 18 & 22 & 0.00688 & 0.00656 & 0.01013 & 15 \\
\hline 12021 & 24 & 633 & 20 & 24 & 0.00125 & 0.00026 & 0.00891 & 1 \\
\hline 13380 & 7 & 686 & 20 & 24 & 0.00814 & 0.00159 & 0.01882 & $1,1,1$ \\
\hline 14765 & 3 & 817 & 20 & 24 & 0.00462 & 0.00565 & 0.01245 & 1,1 \\
\hline 15743 & 1 & 530 & 16 & 24 & 0.01372 & 0.00931 & 0.02686 & $1,10,10$ \\
\hline 17483 & 4 & 491 & 20 & 24 & 0.00412 & 0.00750 & 0.01211 & 1,5 \\
\hline $\mathrm{ACL}$ & 27 & 372 & 16 & 24 & 0.00690 & 0.00657 & 0.01384 & 1 \\
\hline AK & 17 & 707 & 18 & 24 & 0.00960 & 0.00602 & 0.01231 & $2,61,61,61$ \\
\hline MPP & 4 & 216 & 18 & 22 & 0.00732 & 0.00459 & 0.00657 & 1 \\
\hline $\mathrm{RHO}$ & 12 & 779 & 16 & 22 & 0.00090 & 0.00023 & 0.01214 & 1 \\
\hline TGFB2-I5 & 3 & 518 & 18 & 24 & 0.00703 & 0.00032 & 0.01099 & \\
\hline ABCA1 & Z & 281 & 17 & 13 & 0 & 0.01058 & 0.01122 & \\
\hline \multirow[t]{2}{*}{ ADAMTS6 } & Z & 529 & 17 & 13 & 0.00390 & 0.00210 & 0.01408 & 1,1 \\
\hline & & & & Mean & 0.00546 & 0.00536 & 0.01307 & \\
\hline
\end{tabular}

Mclr

11

590

14

22

0.0027

0.00164

0.00581

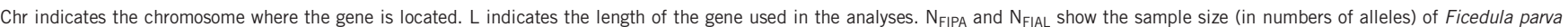
and $F$. albicilla, respectively, in each gene. $\pi$ indicates the nucleotide diversity of each species. Dxy indicates the nucleotide divergence between the two species. Indel length shows the length of individual indel in the corresponding gene.

aitochondrial DNA 


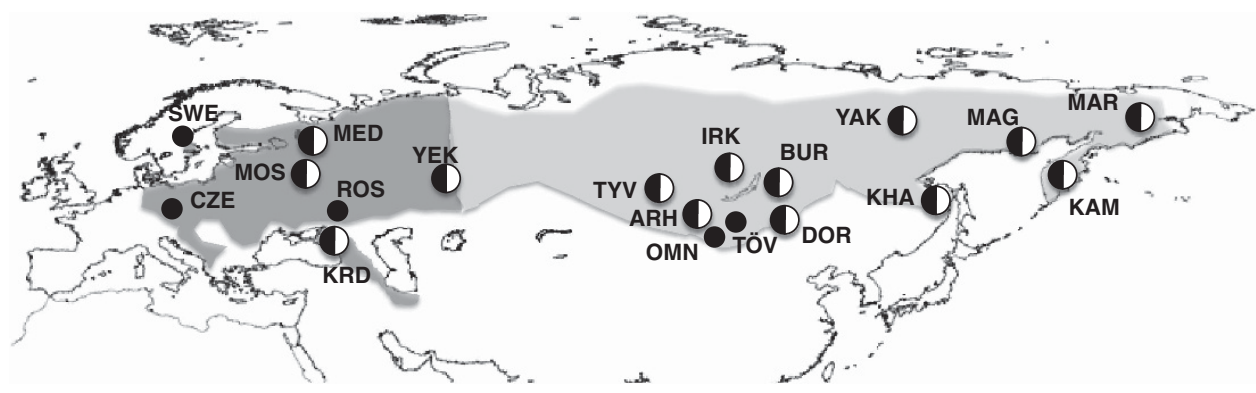

Figure 1 Breeding ranges of $F$. parva (dark gray) and $F$. albicilla (light gray) and distribution of samples sequenced for mtDNA and/or nuclear genes. Black circles indicate the localities where sample(s) were sequenced for mtDNA. Circles with black and white colors indicate the localities where sample(s) were sequenced for both mtDNA and nuclear genes. ARH indicates Arhangay, BUR indicates Buryatiya, CZE indicates Czech Republic, DOR indicates Dornod, IRK indicates Irkutskaya, KAM indicates Kamchatka, KHA indicates Khabarov, KRD indicates Krasnodar, MAG indicates Magadan, MAR indicates Markovo, MED indicates Medvedevo, MOS indicates Moscow, OMN indicates Omnogovi, ROS indicates Rostov, SWE indicates Sweden, TYV indicates Tyva, TÖV indicates Töv, YAK indicates Yakutiya and YEK indicates Yekaterinburg. Information on the numbers of samples sequenced for each gene at each locality is given in Supplementary Table 1.

The phases of these ambiguous sites were resolved using Clark's (1990) parsimony algorithm.

Intra-locus recombination rates were estimated using a Bayesian method (Li and Stephens, 2003) implemented in PHASE 2.1.1. A background recombination rate and the recombination rates between two polymorphic sites were estimated and portrayed as 5000 sampled points from the posterior distribution. The factors by which the estimated recombination rates exceed the background rate were calculated, and the upper and lower bounds of $95 \%$ credible intervals of these values were recorded. If one or more estimated values were significantly larger than one, it meant that there was significant recombination occurring between the corresponding sites (Hung et al., 2012). Otherwise, we assumed no intra-locus recombination.

DnaSP 5 (Librado and Rozas, 2009) was used to compute nucleotide diversity ( $\pi$; Nei, 1987) for each species and nucleotide distance (D; Nei, 1987) between the two species for each gene.

\section{Allele networks}

NETWORK 4.5.1.6 (fluxus-engineering.com) was used to generate a minimum spanning allele network for each nuclear gene to reveal the pattern of divergence between $F$. parva and F. albicilla.

\section{Demographic and divergence history}

The program IMa (Hey and Nielsen, 2007) was used to estimate the effective population sizes of two species and their common ancestor, migration rates and divergence times between the two species. A minimum of $2 \times 10^{7}$ steps after a burn-in of $10^{6}$ steps were performed for each analysis. Plots of trend lines and effective sample size values (ESS >250) were examined to assess convergence in parameter estimates. In addition, at least two independent analyses were performed to assure convergence. The IMa analyses were applied to all 17 introns combined. To convert the scaled demographic parameters of IMa, we calculated the geometric mean of the substitution rates of these 17 loci by multiplying the sequence lengths by $1.35 \times 10^{-9}$ substitutions per site per year for autosomal introns (Ellegren, 2007), and $1.6 \times 10^{-9}$ substitutions per site per year for Z-linked introns (given the mean divergence of Z-linked introns between chicken and turkey is 1.2 times higher than that of autosomal introns (Ellegren 2007). The generation times of the two flycatcher species were set at 2 years because most male birds do not breed until the red throat or breast patch is attained after the second year (Mitrus, 2006). Additional IMa analyses were applied to two other data sets, one contained the 17 introns plus the exon and the other contained 15 introns excluding two introns (00132 and ABCA1), each of which was fixed in one species (see Results).

Although IMa can reconstruct divergence history between sister species by considering multiple demographic parameters, it cannot reveal chronological population dynamics owing to the assumption of no population size change since divergence. To complement the IMa results, we applied the Extended Bayesian Skyline Plot (EBSP) method (Heled and Drummond, 2008) implemented in BEAST v1.6.1 (Drummond and Rambaut, 2007) to estimate population size changes through time. However, EBSP can only trace the history of one species per analysis, not coalescence between sister species. Thus, integrating IMa and EBSP results can provide a better picture of species' divergence and demographic history. The data sets used for the EBSP analyses were the same as those for the IMa analyses. Unlinked substitution estimates based on jModelTest (Posada, 2008) were assigned to each nuclear gene. The strict molecular clock model was used as it generally considered a better fit with analyses at the intraspecific level (Yang, 2006; Bisconti et al., 2011) and it helps to avoid over-parameterization (Thalmann et al., 2013) and thus facilitates convergence of the analyses. A locus that evolves at the fastest rate or is the most divergent was set as the reference locus to calibrate time and population sizes. ADAMTS6, a Z-linked intron, with a substitution rate of $1.6 \times 10^{-9}$ substitutions per site per year and 15743 , which had high $\pi$ values in both species (see Table 1) with a substitution rate of $1.35 \times 10^{-9}$ substitutions per site per year, were used as the reference loci for separate analyses to test for the effects of different reference loci. Each EBSP analysis included a MCMC chain of 500 million steps, which were sampled every 50000 steps, and the first $25 \%$ of the steps were discarded as burn-in. Trace plots were checked using TRACER v 1.5 (Rambaut and Drummond, 2007) to assess convergence in MCMC analyses and three independent runs were performed to assure convergence in estimates. If the $95 \%$ confidence interval (CI) of the number of size-change steps excluded zero, we concluded that significant change(s) in the population size of the focal species had occurred.

\section{Modeling current and historical distributions of the two species} ENMs can predict historical range change of species independent from genetic data. Locality records for breeding birds from each species were obtained from specimen records and ORNIS2 (http://ornis2.ornisnet.org); duplicate records were eliminated. ENMs were constructed using Maxent v3.2.2 (Phillips et al., 2006) for both species at the present, the Last Glacial Maximum (LGM, $21000 \mathrm{ybp}$, CCSM model) and the Last Interglacial (LIG, $120000 \mathrm{ybp}$ ). Climatic data (19 layers) were obtained from the Worldclim bioclimatic database (Hijmans et al., 2005). On the basis of an initial Maxent run of three replicates, climate layers contributing $5 \%$ or more to the model were selected, and the analysis was re-run using these layers. The final niche model was based on the average of five Maxent runs and plotted using DIVA-GIS v7.1.7.2 (Hijmans et al., 2004). The goal was to determine whether the range of F. albicilla was severely contracted at the LGM, which could have led to a bottleneck.

\section{Compound neutrality tests}

The DHEW neutrality test (Zeng et al., 2007), which is robust to demographic history, is a compound test of Tajima's $D$ (Tajima, 1989), Fay and Wu's $H$ (Fay and $\mathrm{Wu}, 2000$ ) and Ewens-Watterson test (Watterson, 1978) and was used to detect positive selection on mtDNA. We applied DHEW tests implemented in the DH program (http://zeng-lab.group.shef.ac.uk/wordpress) to concatenated 
sequences of ND2, Cyt $b$ and COI because the DHEW test relies on segregating sites $(S)$ but $F$. albicilla's ND2 was invariant and its Cytb $(S=2)$ and COI $(S=1)$ provided little variability (see Results). One sequence from each of the two species was used as the outgroup for the other. The significance levels of tests were determined through 50000 simulations.

\section{Multilocus neutrality tests}

Maximum likelihood multilocus Hudson-Kreitman-Aguade (ML-HKA) tests implemented in the MLHKA program (Wright and Charlesworth, 2004) were used to test the neutrality of the mtDNA, the exon and two introns 00132 and ABCA1 that were fixed in F. albicilla and F. parva, respectively. This test is revised from the standard HKA test (Hudson et al., 1987) and provides a test for selection at specific loci (Wright and Charlesworth, 2004). We performed likelihood ratio tests by assigning the remaining 15 introns to a neutral gene set, which was supported by the standard HKA test $(P=0.415)$, against each individual candidate gene (that is, ND2, Cytb, COI, a concatenation of the three mtDNA genes, Mc1r, 00132 and ABCA1). The likelihood ratio tests compared a model considering the candidate gene under selection with a null model assuming that all 16 genes were neutral (degrees of freedom $=1$ ). Each of the two species was used as the outgroup for the other. All runs included 500000 cycles of Markov chains and two independent runs were performed for each model to check for the convergence of likelihood scores.

\section{Testing mtDNA neutrality using simulations}

To test whether the pattern of mtDNA variation deviated from neutral models, while considering demographic history and coalescence variation, we performed neutral coalescence simulations of mtDNA evolution using the program Bayesian Serial SimCoal (Excoffier et al., 2000; Anderson et al., 2005). As Bayesian Serial SimCoal simulates genes not individuals, the ploidy of genes was taken into account. That is, the effective population size of mtDNA $\left(\mathrm{N}_{\mathrm{ef}}\right)$ was $0.5 \times$ the $\mathrm{Ne}$ of diploid individuals $(=0.25 \times$ the $\mathrm{Ne}$ of autosomal genes) assuming a balanced sex ratio, in which the $\mathrm{N}_{\mathrm{e}}$ of diploid individuals was the effective population size estimated from IMa (that is, $\theta / 4 \mu$; J. Hey, pers. comm.). A generation time of 2 years was used for the simulations. The following demographic model incorporating the IMa and EBSP estimates (see Results and Discussion) was used to simulate neutral mtDNA diversity: two species diverged from each other 1.6 million generations ago when the $\mathrm{N}_{\mathrm{ef}}$ of their common ancestor was 370000 , the $\mathrm{N}_{\mathrm{ef}}$ of $F$. parva remained at a similar and constant size, and $F$. albicilla experienced a recent population increase $(\sim 6 \mathrm{X})$ around $210000\left(\sim 0.5 \mathrm{~N}_{\mathrm{ef}}\right)$ generations ago to a $\mathrm{N}_{\mathrm{ef}}$ of 410000 . We assumed an $\sim 5 \mathrm{X}$ population bottleneck in the $\mathrm{N}_{\mathrm{ef}}$ of F. albicilla when it split from $F$. parva, which we suggest provides a reasonable test for detecting departure from a neutral pattern. That is, if neutrality is rejected, factor(s) other than a population bottleneck were the cause of the observed low mtDNA polymorphism of $F$. albicilla. To address uncertainty in the IMa estimates of divergence times and population sizes, we specified simulation parameters as normal distributions, rather than fixed values, with means equal to the values with the highest posterior probability (that is, those described above), covering at least $95 \%$ of the posterior distributions. To maintain a model with a population bottleneck followed by a population expansion in F. albicilla, we used the current $\mathrm{N}_{\mathrm{ef}}$ of F. albicilla to scale all other population sizes and the timing of the population expansion in simulations (Figure 4a). The simulations used parameters based on the 15 introns, instead of all 17 introns, considering the potentially non-neutral patterns of 00132 and ABCA1, although the results of the two data sets were similar (see Results).

The substitution rates of mtDNA used for the simulations were estimated based on the results of this study. As the nucleotide difference between the two species were 0.06854, 0.06069 and 0.05027 in ND2, Cytb and COI, respectively (see Table 1), and the divergence time was about 3.327 million years (IMa estimate based on the 15 introns; see Table 2), the substitution rates of the three mtDNA regions were $0.0103,0.0091$ and 0.0076 per site per million years, respectively. In addition, we used the substitution rates estimated from Hawaiian honeycreepers, which are possibly more applicable to recent divergence (Lerner et al., 2011), for simulation. Those were 0.029, 0.014 and 0.016 per site per million years for ND2, Cytb and COI, respectively.

We simulated 100000 data sets with the same sequence lengths and sample sizes as the empirical data for F. parva and F. albicilla. The $\pi$ values of the simulated data, calculated by Bayesian Serial SimCoal, were compared with those of the empirical data to test whether the mtDNA genetic diversity of the two species departed from neutral expectations.

We also applied the coalescence simulation test to 00132 and ABCAl to examine whether they departed from neutral expectations and whether the patterns of low variation in mtDNA were more extreme than those in the two introns. The $\mathrm{N}_{\mathrm{e}}$ of (autosomal) 00132 and (Z-linked) ABCA1 were assumed as $2 \times$ and $1.5 \times$ the $\mathrm{N}_{\mathrm{e}}$ of diploid individuals, respectively. The substitution rates of 00132 and ABCA1 used for the simulations were estimated using the same approach as that for mtDNA and were 0.0008251 and 0.001686 per site per million years, respectively. Other simulation settings were the same as those for mtDNA.

\section{Test for direct positive selection on mtDNA}

The widely used M-K test (McDonald and Kreitman, 1991) for direct positive selection could not be used in this study owing to the absence or low numbers of nonsynonymous polymorphisms in the mtDNA genes of the two study species. Thus, we devised a test based on the distribution of nonsynonymous and synonymous interspecific differences between transmembrane (TM) and

Table 2 Effective population sizes, migration frequencies and divergence times estimated by IMa

\begin{tabular}{|c|c|c|c|c|c|c|}
\hline & $\mathrm{N}_{\mathrm{e}(\mathrm{FIAL})}$ & $\mathrm{N}_{\mathrm{e}(\mathrm{FIPA})}$ & $\mathrm{N}_{\mathrm{e}(\text { anc })}$ & $\mathrm{M}_{1}$ & $\mathrm{M}_{2}$ & $\mathrm{t}$ \\
\hline \multicolumn{7}{|l|}{15 Introns } \\
\hline HiSmth & 820666 & 742987 & 749649 & 0 & 0 & 3327422 \\
\hline 95Lo & 662270 & 594115 & 451984 & 0 & 0 & 2645477 \\
\hline $95 \mathrm{Hi}$ & 1039143 & 954096 & 1467906 & 0.0399 & 0.0372 & 4432643 \\
\hline \multicolumn{7}{|l|}{17 Introns } \\
\hline HiSmth & 754922 & 654876 & 663658 & 0 & 0 & 3112058 \\
\hline 95Lo & 610042 & 524777 & 389416 & 0 & 0 & 2492660 \\
\hline $95 \mathrm{Hi}$ & 952169 & 837710 & 1259636 & 0.0354 & 0.0330 & 4169719 \\
\hline \multicolumn{7}{|c|}{17 Introns +1 exon } \\
\hline HiSmth & 758884 & 660159 & 653003 & 0 & 0 & 3229241 \\
\hline 95Lo & 616531 & 531170 & 375327 & 0 & 0 & 2569250 \\
\hline $95 \mathrm{Hi}$ & 951031 & 841109 & 1215532 & 0.0334 & 0.0306 & 4232697 \\
\hline
\end{tabular}

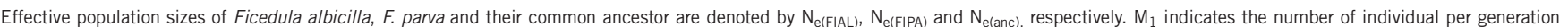

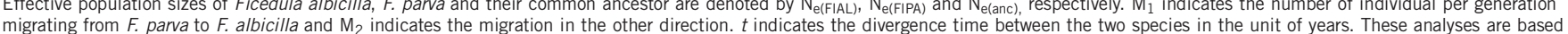

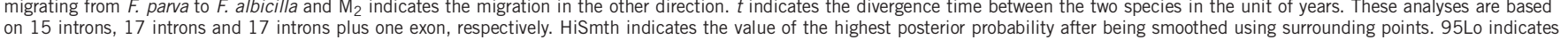
the value to which $2.5 \%$ of the total distribution lies to the left. $95 \mathrm{Hi}$ indicates the value to which $2.5 \%$ of the total distribution lies to the right. 
surface (SF) segments of mtDNA genes (see Wise et al., 1998). If positive selection directly targets mtDNA gene function, nonsynonymous substitutions should be concentrated in TM segments that are subject to more stringent structural and functional constraints (Tourasee and Li, 2000).

The crystallized chicken Cytb protein (3170C.pdb) and bovine heart COI protein (2occA.pdb) were used as templates to model the three-dimensional structures of the two proteins for F. parva and F. albicilla using the SWISSMODEL online server (Arnold et al., 2006; online server at http://swissmodel.expasy.org/). The protein structures and the locations of amino acid replacements were visualized using the program Swiss-PdbViewer v4.02 (Guex and Peitsch, 1997). The TM and SF segments of the Cyt $b$ and COI proteins were predicted using MEMSAT (Jones et al., 1994) through the SWISSMODEL server. We were unable to model the protein structure of ND2 because a crystallized template was not available. Thus, the TM and SF segments of the ND2 protein were predicted using the program HMMTOP v2 (Tusnády and Simon, 2001; the online server at http://www.enzim.hu/ hmmtop/index.html). We also used HMMTOP to predict TM and SF segments for the Cyt $b$ protein and found that the HMMTOP and MEMSAT predictions were very similar and the results of Fisher's exact tests based on the two predictions were the same. Thus, predictions of the two programs were compatible.

We separated the fixed substitutions between the two species into four categories, TM nonsynonymous, SF nonsynonymous, TM synonymous and SF synonymous substitutions, to conduct Fisher's exact tests. The ratio of TM synonymous to SF synonymous substitutions revealed relative mutation rates between TM and SF and was used to test whether the distribution of nonsynonymous substitutions was skewed. We applied this test to the ND2 and Cyt $b$ but not to the COI because there was only one fixed nonsynonymous substitution between the two species.

We further used PROVEAN v.1.1.2 online server (Choi et al., 2012; online server at http://provean.jcvi.org/index.php) to examine whether the nonsynonymous substitutions between the two species' mtDNA have significant effect in protein function. PROVEAN assesses the effect of a substitution based on its resulting change in sequence similarity of a query protein to homologous proteins. The protein database was set as 'NCBI nr, September 2012' and the default delta score threshold $(-2.5)$ was used. The RPOVEAN prediction was applied to the ND2, Cyt $b$ and COI data.

\section{RESULTS}

\section{Genetic diversity of mitochondrial and nuclear genes}

The $\pi$ values for $F$. albicilla (average of ND2, Cytb and $\mathrm{COI}=0.00007 \pm 0.00005$ (s.e.)) were consistently lower than those of $F$. parva (average $=0.00141 \pm 0.00029$ ) in the three mtDNA genes, although the $\pi$ values of F. albicilla were not zero for Cytb (0.00016) or COI (0.00005) as was found for ND2 (Table 1 and Figure 2). There were three Cyt $b$ haplotypes in 25 F. albicilla individuals; however, two singleton haplotypes differed only by one bp from the most frequent one. There were two COI haplotypes in $26 \mathrm{~F}$. albicilla individuals, one of which was one singleton differing by one bp from the most frequent one. In contrast, $\pi$ for the nuclear loci was not significantly different between the two species $(0.00536 \pm 0.00116$ for $F$. albicilla. and $0.00546 \pm 0.00086$ for $F$. parva at introns, $P=0.936$, paired Student's $t$-test; 0.00164 for $F$. albicilla. and 0.0027 for $F$. parva at $M c 1 r$; Figure 2). Two introns had no genetic variation among the samples of one species. Those were 00132 in F. albicilla and ABCA1 in F. parva (Table 1). Most introns (15/17) contained one to six indels ranging from one to $61 \mathrm{bp}$ (Table 1 ). No intra-locus recombination was detected in the 18 nuclear loci.

\section{Allele networks of nuclear genes}

Most nuclear networks (13/18) supported the split of the two species except for 09385,11074 , MPP, TGFB2-I5 and ABCA1, which were unresolved and therefore not conflicting (Supplementary Figure 1).

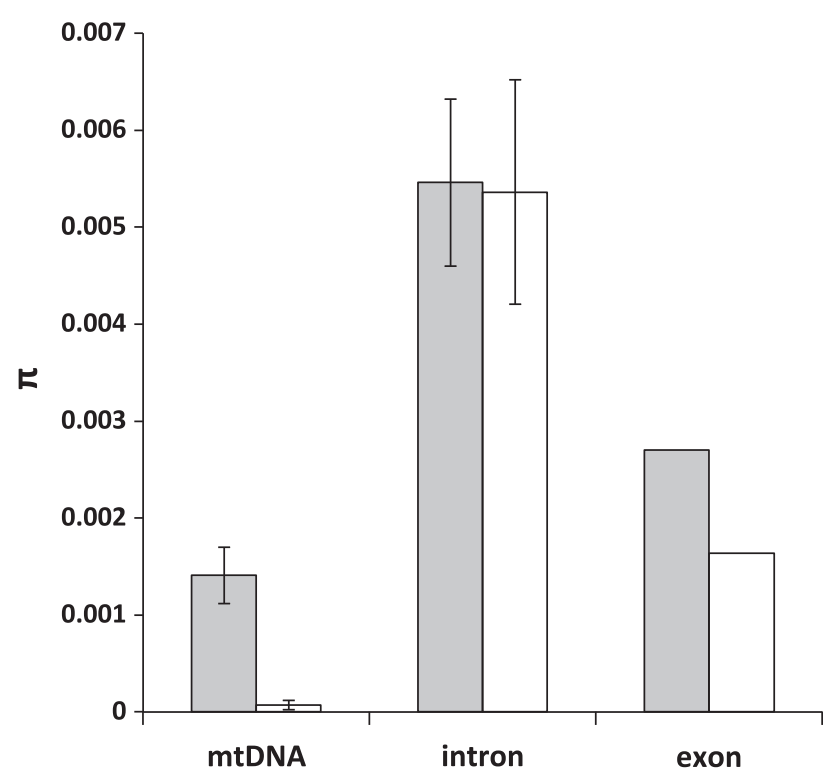

Figure 2 Nucleotide diversity $(\pi)$ of three mtDNA genes, 17 introns and one exon of $F$. parva (gray bars) and F. albicilla (white bars). Shown are the means and SE for mtDNA and introns.

\section{Demographic and divergence history}

The IMa analyses based on the 17 introns suggested similar effective population sizes for $F$. parva $(654876$; 95\% CI $=524777-837710)$ and F. albicilla (754922; 95\% CI =610 042-952 169; Table 2). The effective population size of their common ancestor (663658; $95 \%$ $\mathrm{CI}=389416-1259636)$ was similar to those of the two species. The divergence time between the two species was estimated at 3112058 (95\% CI $=2492660-4169719)$ years ago. There has been no gene flow between the two species (Table 2). The analyses based on the 17 introns plus one exon and 15 introns excluding 00132 and ABCA1 suggested similar histories as those based on the 17 introns (Table 2).

The EBSP analyses, however, suggested that $F$. albicilla experienced a recent population expansion, whereas $F$. parva has had a stable effective population size based on the 17 introns (Figure 3; the $95 \%$ CIs of the number of size-change steps were 1-2 for the former and 0-1 for the latter). The EBSP results based on the three data sets suggested similar history of a population expansion in F. albicilla and a stable population for F. parva (data not shown).

\section{Modeling current and historical distributions of the two species} The ENMs for both species suggested that their ranges were reduced at the LGM compared with the LIG and present (Supplementary Figure 2). However, there was no evidence for a dramatic range contraction during the past 120000 years that would explain the extremely low mtDNA variability in F. albicilla.

\section{Neutrality tests}

The DHEW tests suggested that the pattern of concatenated mtDNA sequences for $F$. albicilla was consistent with positive selection or selective sweeps $(P=0.00034)$ but not for that of $F$. parva $(P=0.12)$.

The ML-HKA tests rejected the neutral model in which the three mtDNA genes were added individually or in concert for both F. albicilla $(P \leqslant 0.000015)$ and $F$. parva $(P=0.0012-0.0222)$, indicating a non-neutral pattern of variation in mtDNA. However, the ML-HKA tests could not reject the neutral models for Mc1r, 00132 and ABCA1 in either species $(P=0.161-0.599)$ except for ABCA1 in 


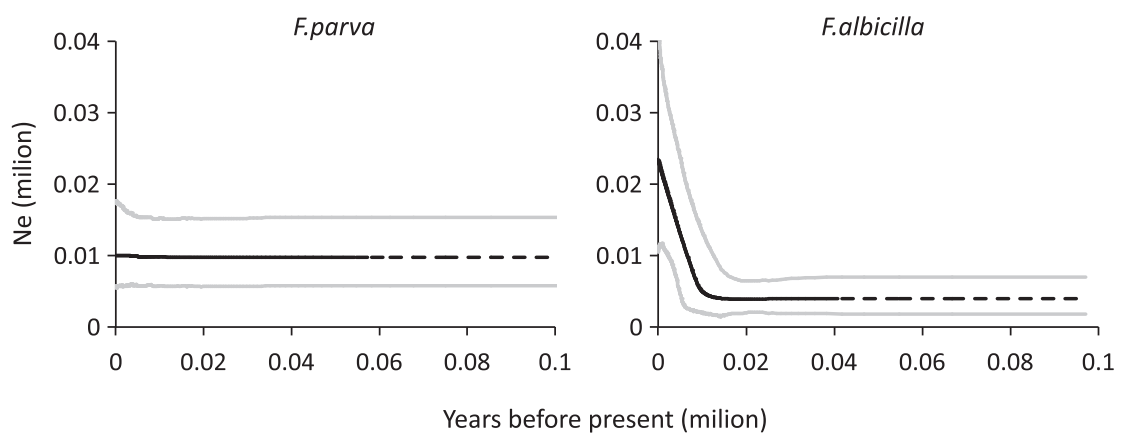

Figure 3 Extended Bayesian Skyline Plots of population sizes of the two study species. Black dashed lines indicate the median values. Gray lines indicate 95\% highest posterior density (HPD). ADAMTS6 is set as the reference locus to calibrate time and population size in this figure.

a

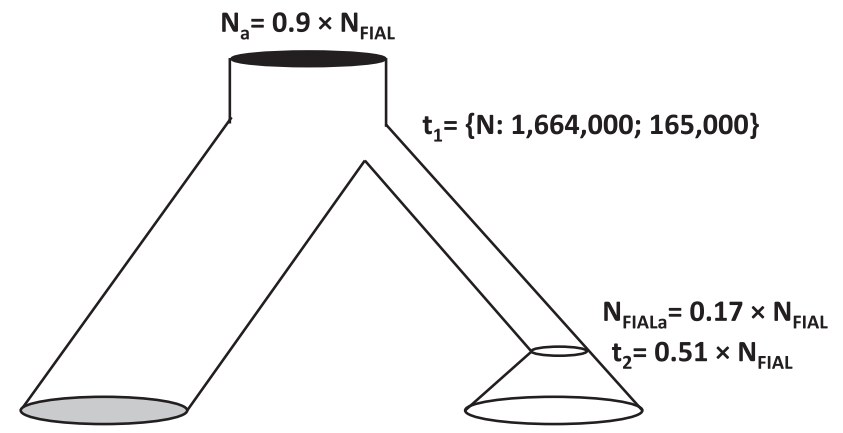

$\mathrm{N}_{\mathrm{FIPA}}=0.9 \times \mathrm{N}_{\mathrm{FIAL}}$

$N_{\text {FIAL }}=\{N: 410,000 ; 35,000\}$

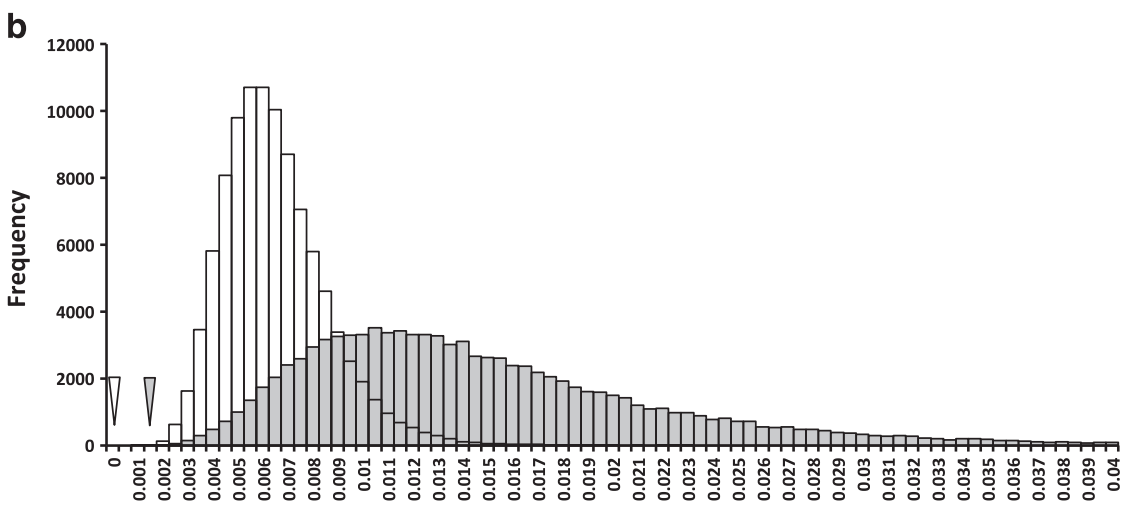

$\pi$

Figure 4 (a) The outline of the coalescence model incorporating the IMa and EBSP estimates based on the 15 introns excluding 00132 and ABCA1. This

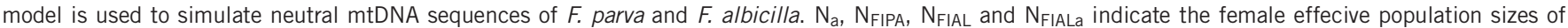
their common ancestor, $F$ parva, $F$ albicilla, and the latter before a population expansion, respectively. $t_{1}$ and $t_{2}$ indicate the divergence time between the two species and the initial time of the population expansion of $F$. albicilla, respectively, in a unit of generations from present. The parameters are set as normal distributions instead of fixed values. The symbol $\{\mathrm{N}: 410000 ; 35000\}$ indicates a normal distribution with a mean of 410000 and a s.d. of 35000 , and $\{\mathrm{N}: 1664000 ; 165000\}$ indicates one with a mean of 1664000 and a s.d. of 165000 . (b) The distributions of nucleotide diversity ( $\pi$ ) values for 100000 neutrally simulated ND2 data sets in F. parva (grey bars) and F. albicilla (white bars). The grey triangle represents the empirical $\pi$ value of ND2 in F. parva and the white one for F. albicilla.

F. parva, for which the neutral model was marginally rejected $(P=0.0494)$.

The results of DHEW and ML-HKA tests collectively suggested that the mtDNA variation of $F$. albicilla departed from neutral expectation, whereas selection was less likely an influence on F. parva.

\section{Testing mtDNA neutrality using simulations}

The comparison of the simulated and empirical data rejected a neutral pattern for the mtDNA of both F. parva and F. albicilla. The empirical $\pi$ value of ND2 in F. albicila was lower than the range of $\pi$ values of the simulated neutral data and that in F. parva was lower than the $99.99 \%$ range of the simulated $\pi$ values based on the substitution rates estimated in this study (Figure 4b); the empirical $\pi$ values of ND2 in both species were also lower than the range of the simulated $\pi$ values based on the substitution rate of Lerner et al. (2011) (data not shown). The same result was found for the Cytb and COI data for both species (data not shown). 
Table 3 Numbers of fixed nonsynonymous and synonymous substitutions between Ficedula parva and $F$. albicilla at the transmembrane and surface segments of ND2 and Cytb.

\begin{tabular}{lcccccc}
\hline Fixed substitution & \multicolumn{3}{c}{ ND2 } & & \multicolumn{3}{c}{ Cytb } \\
\cline { 2 - 3 } \cline { 5 - 7 } \cline { 5 - 7 } & Transmembrane & Surface & & Transmembrane & Surface \\
\hline Nonsynonymous & 12 & 1 & & 8 & 1 \\
Synonymous & 30 & 24 & & 30 & 21 \\
FET probability & \multicolumn{2}{c}{0.012} & & & 0.083 & \\
\hline
\end{tabular}

Abbreviation: FET, Fisher's exact one-tailed test.

FET probability indicates the probability of Fisher's exact one-tailed test.

The simulation results rejected a neutral pattern for ABCA1 in both species with lower confidence than those for mtDNA but could not reject a neutral pattern for 00132 in either species. The empirical $\pi$ values of $F$. albicilla and $F$. parva for ABCA1 were out of the $95 \%$ range but were within the $99 \%$ range of the simulated ones (Supplementary Figure 3). Noticeably, the former was located in the upper tail of the range (that is, non-neutrally high polymorphism) and the latter was in the lower tail of the range (that is, non-neutrally low polymorphism). In contrast, the empirical $\pi$ values of both species for 00132 were within the $90 \%$ range of the simulated ones (Supplementary Figure 3).

\section{Test for direct positive selection on mtDNA}

Nonsynonymous substitutions tended to occur in the TM regions (12 TM:1 SF) of the ND2 protein more than synonymous ones between the two study species (30 TM:24 SF; $P_{\text {one-tail }}=0.012$, Fisher's exact test; Table 3). We also found more TM nonsynonymous substitutions $(n=8)$ than SF nonsynonymous substitutions $(n=1)$ in the Cytb protein (Table 3; Supplementary Figure 4), although the TM:SF ratios of nonsynonymous and synonymous substitutions were not significantly different $\left(P_{\text {one-tail }}=0.083\right.$; Table 3$)$.

PROVEAN predicted that three TM nonsynonymous substitutions in ND2 between the two species, Threonine (F. albicilla) to Isoleucine (F. parva) at the 18th codon, Isoleucine (F. albicilla) to Threonine (F. parva) at the 19th codon and Threonine (F. parva) to Alanine (F. albicilla) at the 152th codon could change protein function. No nonsynonymous substitutions in the Cytb and COI data were predicted to have a functional effect.

\section{DISCUSSION}

Contrasting genetic diversity between mtDNA and nuclear DNA (ncDNA)

One explanation for the lack of polymorphism in the ND2 of F. albicilla is that Zink et al. (2008) inadvertently sequenced pseudogenes. However, mtDNA polymorphism in F. albicilla was consistently lower than that in F. parva across ND2, Cytb and COI. Therefore, it is unlikely that nuclear copies of mtDNA were sequenced in all three regions because they were well separated throughout the mtDNA genome. Furthermore, (1) the PCR products for ND2 derived from four primer pairs returned the same sequencing results, (2) there were no indels or stop codons found in ND2 and (3) correct sister relationships between the two species and between their clade and the other clade containing F. hypoleuca, F. albicollis, F. speculigera and $F$ semitorquata were obtained based on the ND2 data (results not shown). Together, these observations support the mitochondrial authenticity of the ND2 data.
In contrast to the mtDNA data, the ncDNA polymorphism levels and the estimated $\mathrm{N}_{\mathrm{e}}$ based on multiple nuclear genes were similar between F. albicilla and F. parva. Therefore, difference in $\mathrm{N}_{\mathrm{e}}$ is not an explanation for the lower level of mtDNA polymorphism in F. albicilla. However, the EBSP revealed a recent population expansion in F. albicilla but not F. parva. Thus, the effect of historical population changes on mtDNA patterns should be considered. In addition, this result can be confounded by large standard errors in mtDNA diversity due to stochastic coalescence processes (Edwards and Bensch, 2009). Thus, repeated coalescence simulations based on population history, which we did in this study, are needed to confirm whether these striking patterns depart from neutral expectation.

\section{Departure from neutral patterns in mtDNA}

The seeming disagreement between the EBSP and IMa estimated histories could be caused by the simplified model of IMa. On the other hand, the EBSP estimate of population size and expansion timing varied with the choice of a reference locus. For example, the current effective population size of $F$. albicilla was estimated as 23500 when ADAMTS6 was assigned as the reference locus, whereas the estimated size was 867000 when 15743 was used. The initial time of the F. albicilla population expansion was around 12000 and 390000 years ago when ADAMTS6 and 15743 were the reference loci, respectively. Nevertheless, the extent of population increase $(\sim 6 \mathrm{X})$ and the ratios of the initial time of the population expansion to the current population size $(\sim 0.5)$ were consistent across different reference loci. Thus, we incorporated the IMa and EBSP results for coalescence simulations to take the advantage of precise IMa estimates, which are based on the geometrical mean of substitution rates for all loci, and explicit demographic fluctuation revealed by EBSP (see Materials and Methods).

Neutral coalescence simulations predicted slightly lower $\pi$ values in F. albicilla than for F. parva; however, the difference was not significant (Figure 4b). Thus, different demographic histories are unable to explain the different levels of mtDNA diversity between the two species.

The low mtDNA diversity in F. albicilla departed from the neutral expectation based on our simulations. Therefore, the modeled bottleneck cannot explain such low mtDNA variation observed in F. albicilla. However, the bottleneck (with a magnitude of $\sim 5 \mathrm{X}$ ) we modeled is much less severe than typically expected for great genetic effect (Nei et al., 1975), although the magnitude of population reduction we assumed was consistent with our multilocus estimates. It explains the observed mtDNA results of the DHWE and ML-HKA tests, which suggested that selection is likely responsible for the low mtDNA diversity of $F$. albicilla.

The observed mtDNA diversity of $F$. parva was also significantly lower than the neutral expectation, which is supported by the MLHKA test but not the DHEW test. The inconsistent results between the DHEW test and the ML-HKA test or the coalescence simulation approach could be due to the strengths and sensitivities of the tests. In particular, the DHEW test can be more conserved because it integrates multiple tests. The mtDNA diversity of $F$. parva ( $\pi_{\mathrm{S}}$ (synonymous nucleotide polymorphism) $=0.0047 \pm 0.0006, \pi_{\mathrm{N}}$ (nonsynonymous nucleotide polymorphism $)=0.0003 \pm 0.0002$ ) is lower than other avian species $\left(\pi_{\mathrm{S}}=0.02-0.06, \pi_{\mathrm{N}}=0.0015-0.005\right.$ for 72 avian species; see Hughes and Hughes, 2007). Of course, the mtDNA diversity of F. albicilla is even lower. The mtDNA variation patterns of the two flycatchers were anomalously low for birds.

The fact that the two introns ABCA1 and 00132 were fixed in F. parva and F. albicilla, respectively, points to the possibility that the 
low mtDNA variation in the two species could happen by chance. The coalescence simulation approach and the ML-HKA test could marginally reject a neutral model for ABCA1 but could not for 00132 . Although the results are mixed, they suggest that the magnitude of departure from neutral expectation for mtDNA is more extreme than that for the two introns, and unlikely to happen by chance.

\section{Tests for positive selection targeting mtDNA}

The TM segments of ND2 accumulated more nonsynonymous substitutions than the SF segments between the two flycatchers. This result differs from some studies. Kerr (2011) found that nonsynonymous substitutions were equally distributed between helix (primarily TM) and loop (primarily SF) sites based on the COI data of 43 avian species across 12 orders; he found no evidence for a selective sweep in the avian COI data. Tourasse and Li (2000) reported that the TM segments of proteins accumulated fewer nonsynonymous substitutions than the SF segments in mammals across six orders and suggested that the TM segments were subject to more stringent structural constraint. The contrasting patterns between this study and others imply that the ND2 protein of either F. parva or F. albicilla or both may be targeted by positive selection. Although relaxed purifying selection can also lead to elevated nonsynonymous substitutions in one or both types of segments, it should not lead to extremely low levels of genetic variation. In addition, coalescence analyses suggested a stable population size for $F$. parva and a recent or ongoing population expansion in $F$. albicilla, which impede relaxation of purifying selection (Ohta, 1973). Thus, positive selection is more likely to be the explanation for the observed pattern rather than relaxed purifying selection.

Furthermore, three TM nonsynonymous substitutions in the ND2 between the two study species were predicted to cause functional change in the protein. Two of the substitutions $\left(\mathrm{Thr}^{18}\right.$ to $\mathrm{Ile}^{18}$; $\mathrm{Ile}^{19}$ to $\mathrm{Thr}^{19}$ ) were from F. albicilla to $F$. parva and the third one ( $\mathrm{Thr}^{152}$ to $\mathrm{Ala}^{152}$ ) was from $F$. parva to $F$. albicilla. All three substitutions change the polarity of amino acids. $\mathrm{Ile}^{18}, \mathrm{Thr}^{19}$ and $\mathrm{Ala}^{152}$ are unique derived states, whereas $\mathrm{Thr}^{18}$, $\mathrm{Ile}^{19}$ and $\mathrm{Thr}^{152}$ are ancestral states found in most of the Ficedula species whose ND2 sequences are available in Genbank (Supplementary Table 2). The results imply positive selection on novel advantageous mutations in each of the two study species. Additional studies on mechanistic links between phenotypic or physiological changes and genetic substitutions and consequent ecological interactions are warranted. Despite the plausible evidence found here supporting positive selection directly targeting the mtDNA ND2 gene, alternative hypotheses such as mitochondrialnuclear coadaptation (Gershnoi et al., 2009) and selective sweeps caused by linkage to either other mtDNA genes (Oliveira et al., 2007) or the $\mathrm{W}$ chromosome due to shared segregation process (Berlin et al., 2007) are worthy of further investigation.

\section{The phylogeography of the two flycatchers based on selected mtDNA, ncDNA and ENMs}

The multilocus analyses suggested that $F$. albicilla and F. parva, which had long been considered conspecific, diverged from each other around 3 million years ago. The well-sorted nuclear genes (Supplementary Figure 1) supported their taxonomic split, which was based on distinguishable plumage characters (Cramp and Perrins, 1993) and highly diverged mtDNA (Zink et al., 2008).

The ENMs for the two species exhibit characteristic features of northern hemisphere species that experienced southward range retractions during the LGM, followed by northward advance, despite the lack of major ice sheets occurring in northern Asia (Adams, 2002).
The magnitude of range contraction at the LGM relative to the LIG and the present is not commensurate with the near absence of mtDNA variation in F. albicilla. Although the timing of the population fluctuations suggested by the genetic analyses apparently predate the range fluctuations since the LIG, the ncDNA results suggest no distinct differences in population history between the two species, which the ENMs confirm for at least the past 120000 years.

The ENMs help to reconstruct the selection-driven history of the mtDNA. The time from introduction to fixation of a favored haplotype in a population is $\sim 2 \ln (2 N) / s$, where $N$ is the population size of the focal gene and $s$ is the selection coefficient (Stephan et al., 1992). Assuming that $s$ acting on F. albicilla's mtDNA is within the range of those on color polymorphisms across diverse taxa, 0.0130.67 (Hoekstra et al., 2004), the fixation time is 41-2095 generations if $N$ is set as the current effective population size (410 000), or 35-1822 generations if the $N$ is set as that before the recent population expansion (70000; Figure 4a). Thus, the dominant haplotype in the mtDNA of $F$. albicilla would have arisen and spread when this species was experiencing a post-LGM population expansion. It is consistent with the theoretical prediction that the fixation frequencies of advantageous mutations increase with population sizes (Meiklejohn et al., 2007).

Therefore, demographic and selective forces might have worked in concert to lead to the unique pattern in which there is almost no mtDNA variation in F. albicilla, across an area of $5000 \mathrm{~km}$. One has to assume that if a highly advantageous mutant arose, presumably in a single female, a high level of gene flow was needed to lead to its rapid spread over a maximum of 2095 generations ( $c a .4190$ years). Thus to explain the current distribution of mtDNA variation in F. albicilla, both a high selection coefficient and a high level of gene flow are inferred.

\section{CONCLUSION}

The simulations and ML-HKA and DHEW tests indicate that the unusually low mtDNA variation of $F$. albicilla has mainly been driven by selection and that demographic history alone has had little impact, although high levels of gene flow are required to explain the distribution of the common mtDNA haplotype. The mtDNA variation of $F$. parva has also been reduced by selection, but to a lesser extent compared with F. albicilla. It is possible that selection strength is lower or selection operates too recently to reach fixation in the mtDNA variation of $F$. parva. The TM-skewed nonsynonymous substitution distribution and the functional change caused by TM amino-acid substitutions in ND2 imply that positive selection has drastically reduced mtDNA variation, although the possibility that selection on linked (such as other mtDNA genes) or functionally associated genes causes their low mtDNA variation cannot be completely ruled out. This study presents a clear case that demonstrates how natural selection can shape genetic variation within and among populations.

A number of published studies (for example, Edwards and Bensch, 2009; Galtier et al., 2009) have claimed that natural selection on $\mathrm{mtDNA}$ is sufficiently pervasive to prevent phylogeographic inference. We found strong evidence of selection on the mtDNA of these flycatchers, especially F. albicilla. However, we suggest that three important inferences can be obtained from the mtDNA pattern, even if it was influenced by natural selection. First, the mtDNA data corroborate the species limits, which are revealed in morphology and nuclear genes. Second, the existence of the same haplotype across a wide area means that there are no barriers to dispersal, because even if positive selection was the cause of the lack of variability, the favored 
haplotype cannot occur where individuals do not disperse. Third, the lack of variation suggests that the event leading to the homogenization of the mitochondrial genome was recent and rapid (Przeworkski, 2002), as otherwise one would expect a series of random point mutations found idiosyncratically across the range. Thus, although the level of variability observed in the mtDNA of $F$. albicilla is anomalously low, several important evolutionary inferences could be obtained. If we only had nuclear loci, this complex history would not be apparent. Several recent studies compared mtDNA and nuclear genes, discovering intriguing processes of population differentiation, local adaptation or unexpected mtDNA dynamics (for example, Cheviron and Brumfield, 2009; Ribeiro et al., 2011; Pavlova et al., 2013). Accumulating multilocus studies have shown that when mtDNA is under selection, adding this marker will gain a more comprehensive phylogeographic picture. Lastly, a few persuasive exceptions should not discount the fact that mtDNA often reveals geographically and taxonomically important patterns, especially among recently isolated taxa (Zink and Barrowclough, 2008; McKay and Zink, 2010).

\section{DATA ARCHIVING}

Data deposited in GenBank: accession numbers KJ362224-KJ363064 and in the Dryad repository: doi:10.5061/dryad.m4sn8.

\section{CONFLICT OF INTEREST}

The authors declare no conflict of interest.

\section{ACKNOWLEDGEMENTS}

We are grateful to S Rowher, S Drovetski and A Pavlova for their contribution in specimen collection and preparation. M Westberg provided laboratory assistance, $\mathrm{T}$ Rodrigues aided in map-making and $\mathrm{S}$ Weller helped to improve the manuscript. We thank L Dong for advising F. albicilla occurrence data collection. Support came from NSF (DEB 0919494). The University of Minnesota Supercomputing Institute provided critical assistance with the computations.

Adams JM (2002). Global land environments since the last interglacial. Available at. http// www.esd.ornl.gov/projects/qen/merc/html.

Anderson CNK, Ramakrishnan U, Chan YL, Hadly EA (2005). Serial Simcoal: a population genetic model for data from multiple populations and points in time. Bioinformatics 21: 1733-1734.

Arnold K, Bordoli L, Kopp J, Schwede T (2006). The SWISS-MODEL Workspace: web-based environment for protein structure homology modeling. Bioinformatics 22 195-201.

Backström N, Brandström M, Gustafsson L, Qvarnström A, Cheng H, Ellegren H (2006). Genetic mapping in a natural population of collared flycatchers (Ficedula albicollis): conserved synteny but gene order rearrangements on the avian z chromosome. Genetics 17: 377-386.

Backström N, Fagergerg S, Ellegren H (2008). Genomics of natural bird populations: a gene-based set of reference markers evenly spread across the avian genome. $\mathrm{Mol} E \mathrm{Col}$ 17: 964-980.

Bazin E, Glémin S, Galtier N (2006). Population size does not influence mitochondrial genetic diversity in animals. Science 312: 570-572.

Bensch S, Irwin DE, Irwin JH, Kvist L, Åkesson S (2006). Conflicting patterns of mitochondrial and nuclear DNA diversity in Phylloscopus warblers. Mol Ecol 15: 161-171.

Berlin S, Tomaras D, Charlesworth B (2007). Low mitochondrial variability in birds may indicate Hill-Roberson effects on the W chromosome. Heredity 99: 389-396.

Bisconti R, Canestrelli D, Colangelo P, Nascetti G (2011). Multiple lines of evidence for demographic and range expansion of a temperate species (Hyla sarda) during the last glaciation. Mol Ecol 20: 5313-5327.

Cheviron ZA, Brumfield RT (2009). Migration-selection balance and local adaptation of mitochondrial haplotypes in rufous-collared sparrows (Zonotrichia capensis) along an elevational gradient. Evolution 63: 1593-1605

Choi Y, Sims GE, Murphy S, Miller JR, Chan AP (2012). Predicting the functional effect of amino acid substitutions and indels. PLos One 7: e46688.

Clark AG (1990). Inference of haplotypes from PCR-amplified samples of diploid populations. Mol Biol Evol 7: 111-122.
Cramp S, Perrin C (1993). Handbook of the Birds of Europe, the Middle East and North Africa: the Birds of the Western Palearctic: Flycatchers to Shrikes, Vol. 8. Oxford University Press: Oxford, UK.

Drummond AJ, Rambaut A (2007). BEAST: Bayesian evolutionary analysis by sampling trees. BMC Evol Biol 7: 214.

Edwards S, Bensch S (2009). Looking forwards or looking backwards in avian phylogeography? A comment on Zink and Barrowclough 2008. Mol Ecol 18: 2930-2933.

Ellegren H (2007). Molecular evolutionary genomics of birds. Cytogenet Genome Res 117 : $120-130$.

Excoffier L, Novembre J, Schneider S (2000). SIMCOAL: a general coalescent program for simulation of molecular data in interconnected populations with arbitrary demography. J Hered 91: 506-509.

Fay JC, Wu C-I (2000). Hitchhiking under positive Darwinian selection. Genetics 155 $1405-1413$

Friesen VL, Congdon BC, Kidd MG, Birt TP (1999). Polymerase chain reaction (PCR) primers for the amplification of five nuclear introns in vertebrate. $\mathrm{Mol} E \mathrm{EO} / 8$ 2147-2149.

Galtier N, Nabholz B, Glémin S, Hurst GDD (2009). Mitochondrial DNA as a marker of molecular diversity: a reappraisal. $\mathrm{Mol}$ Ecol 18: 4541-4550.

Gershoni M, Templeton AR, Mishmar D (2009). Mitochondrial bioenergetics as a major motive force of speciation. BioEssays 31: 642-650.

Guex N, Peitsch MC (1997). SWISS-MODEL and the Swiss-PdbViewer: an environment for comparative protein modeling. Electrophoresis 18: 2714-2723.

Hahn MW (2008). Toward a selection theory of molecular evolution. Evolution 62 255-265.

Heled J, Drummond AJ (2008). Bayesian inference of population size history from multiple loci. BMC Evol Biol 8: 289

Hey J, Nielsen R (2007). Integration within the Felsenstein equation for improved Markov chain Monte Carlo methods in population genetics. Proc Natl Acad Sci USA 104 2785-2790.

Hijmans RJ, Guarino L, Bussink C, Mathur P, Cruz M, Barrentes I et al. (2004). DIVA-GIS Vsn. 7.1.7. A geographic information system for the analysis of species distribution data. Available at. http://www.diva-gis.org

Hijmans RJ, Cameron SE, Parra JL, Jones PG, Jarvis A (2005). Very high resolution interpolated climate surfaces for global land areas. Int J Climatol 25: 1965-1978.

Hoekstra HE, Drumm KE, Nachman MW (2004). Ecological genetics of adaptive colo polymorphism in pocket mice: geographic variation in selected and neutral genes. Evolution 58: 1329-1341.

Hudson RR, Kreitman M, Aguadé M (1987). A test of neutral molecular evolution based on nucleotide data. Genetics 116: 153-159.

Hughes AL (2007). Looking for Darwin in all the wrong places: the misguided quest for positive selection at the nucleotide sequence level. Heredity 99: 364-373.

Hughes AL, Hughes AK (2007). Coding sequence polymorphism in avian mitochondrial genomes reflects population histories. Mol Ecol 16: 1369-1376.

Hung C-M, Drovetski SV, Zink RM (2012). Multilocus coalescence analyses support a mtDNA-based phylogeographic history for widespread Palearctic passerine bird, Sitta europaea. Evolution 66: 2850-2864.

Jones DT, Taylor WR, Thornton JM (1994). A model recognition approach to the prediction of all-helical membrane protein structure and topology. Biochemistry 33 : 3038-3049.

Kerr KCR (2011). Searching for evidence of selection in avian DNA barcodes. Mol Ecol Resour 11: 1045-1055.

Kimball RT, Braun EL, Barker FK, Bowie RCK, Braun MJ, Chojnowski JL et al. (2009). A well-tested set of primers to amplify regions spread across the avian genome. $\mathrm{Mo}$ Phyl Evol 50: 654-660.

Kimura M (1983). The Neutral Theory of Molecular Evolution. Cambridge University Press: Cambridge, UK.

Lerner HRL, Meyer M, James HF, Hofreiter M, Fleischer RC (2011). Multilocus resolution of phylogeny and timescale in the extant adaptive radiation of Hawaiian Honeycreepers. Curr Biol 21: 1-7.

Li N, Stephens M (2003). Modeling linkage disequilibrium, and identifying recombination hotspots using SNP data. Genetics 165: 2213-2233.

Librado P, Rozas J (2009). DnaSP v5: A software for comprehensive analysis of DNA polymorphism data. Bioinformatics 25: 1451-1452.

MacDougall-Shackleton EA, Blanchard L, Gibbs HL (2003). Unmelanized plumage patterns in old world leaf warblers do not correspond to sequence variation at the Melanocortin-1 Receptor locus (MC1R). Mol Biol Evol 20: 1675-1681.

Maynard-Smith J, Haigh J (1974). The hitch-hiking effect of a favourable gene. Genet Res 23: $23-35$.

McDonald JH, Kreitman M (1991). Adaptive protein evolution at the Adh locus in Drosophila. Nature 351: 652-654.

McKay BD, Zink RM (2010). The causes of mitochondrial DNA gene tree paraphyly in birds. Mol Phylo Evol 54: 647-650.

Meiklejohn CD, Montooth KL, Rand DM (2007). Positive and negative selection on the mitochondrial genome. Trends Genet 23: 259-263.

Mitrus $C$ (2006). The influence of male age and phenology on reproductive success of the red-breasted flycatcher (Ficedula parva Bechst.). Ann Zool Fennici 43: 358-365.

Nei M (1987). Molecular Evolutionary Genetics. Columbia University Press: New York, NY, USA.

Nei M, Maruyama T, Chakraborty R (1975). The bottleneck effect and genetic variability in populations. Evolution 29: 1-10.

Ohta T (1973). Slightly delterious mutant substitutions in evolution. Nature 246: 96-98. 
Oliveira DCS, Raychoudhury R, Lavrov DV, Werren JH (2007). Rapidly evolving mitochondrial genome and directional selection in mitochondrial genes in the parasitic wasp Nasonia (Hymenoptera: Pteromalidae). Mol Biol Evol 25: 2167-2180.

Pavlova A, Amos JN, Joseph L, Loynes K, Austin JJ, Keogh J et al. (2013). Perched at the mito-nuclear crossroads: divergent mitochondrial lineages correlate with environment in the face of ongoing nuclear gene flow in an Australian bird. Evolution 67: 3412-3428.

Phillips SJ, Anderson RP, Schapire RE (2006). Maximum entropy modeling of species geographic distribution. Ecol Model 19: 231-259.

Posada D (2008). jModelTest: phylogenetic model averaging. Mol Biol Evol 25: 1253-1256.

Primmer CR, Borge T, Lindell J, Sætre G-P (2002). Single-nucleotide polymorphism characterization in species with limited available sequence information: high nucleotide diversity revealed in the avian genome. Mol Ecol 11: 603-612.

Przeworski M (2002). The signature of positive selection at randomly chosen loci. Genetics 160: 1179-1189.

Rambaut A, Drummond AJ (2007). Tracer v1.4, Available from. http://beast.bio.ed.ac.uk/ Tracer.

Ribeiro ÂM, Lloyd R, Bowie RCK (2011). A tight balance between natural selection and gene flow in a southern African arid-zone endemic bird. Evolution 65: 3499-3514.

Rosenberg N, Nordborg M (2002). Genealogical trees, coalescent theory and the analysis of genetic polymorphisms. Nat Rev Genet 3: 380-390.

Shapiro LH, Dumbacher JP (2001). Adenylate Kinase Intron 5: a new nuclear locus for avian systematics. Auk 118: 248-255.

Stephan W, Wiehe THE, Lenz MW (1992). The effect of strongly selected substitutions on neutral polymorphism: analytical results based on diffusion theory. Theor Popul Biol 41: 237-254.
Stephens M, Smith N, Donnelly P (2001). A new statistical method for haplotype reconstruction from population data. Am J Hum Genet 68: 978-989.

Sousa-Santos C, Robalo JI, Collarrs-Pereira M-J, Almada VC (2005). Heterozygous indels as useful tools in the reconstruction of DNA sequences and in the assessment of ploidy level and genomic constitution of hybrid organisms. DNA Seq 16: 462-467.

Tajima F (1989). Statistical method for testing the neutral mutation hypothesis by DNA polymorphism. Genetics 123: 585-595.

Thalmann O, Shapiro B, Cui P, Schuenemann VJ, Sawyer SK, Greenfield DL et al. (2013). Complete mitochondrial genomes of ancient canids suggest a European origin of domestic dogs. Science 342: 871-874.

Tourasse NJ, Li W-H (2000). Selective constraints, amino acid composition, and the rate of protein evolution. Mol Biol Evol 17: 656-664.

Tusnády GE, Simon I (2001). The HMMTOP transmembrane topology prediction server. Bioinformatics 17: 849-850.

Watterson GA (1978). The homozygosity test of neutrality. Genetics 88: 405-417.

Wise CA, Sraml M, Easteal S (1998). Departure from neutrality at the mitochondrial NADH dehydrogenase subunit 2 gene in humans, but not in chimpanzees. Genetics 148: 409-421.

Wright SI, Charlesworth B (2004). The HKA test revisited: A Maximum-Likelihood-Ratio Test of the standard neutral model. Genetics 168: 1071-1076.

Yang Z (2006). Computational Molecular Evolution. Oxford University Press: Oxford, UK. Zeng K, Suhua S, Wu C-I (2007). Compound tests for the detection of hitchhiking under positive selection. Mol Biol Evol 24: 1898-1908.

Zink RM, Barrowclough GF (2008). Mitochondrial DNA under siege in avian phylogeography. Mol Ecol 17: 2107-2121.

Zink RM, Pavlova A, Drovetski SV, Rohwer S (2008). Mitochondrial phylogeographies of five widespread Eurasian bird species. J Ornithol 149: 399-413.

Supplementary Information accompanies this paper on Heredity website (http://www.nature.com/hdy) 